\title{
Generalized Difference $\lambda$-Sequence Spaces Defined by Ideal Convergence and the Musielak-Orlicz Function
}

\author{
Awad A. Bakery ${ }^{1,2}$ \\ ${ }^{1}$ Department of Mathematics, Faculty of Science and Arts, King Abdulaziz University (KAU), P.O. Box 80200, \\ Khulais 21589, Saudi Arabia \\ ${ }^{2}$ Department of Mathematics, Faculty of Science, Ain Shams University, P.O. Box 1156, Abbassia, Cairo 11566, Egypt
}

Correspondence should be addressed to Awad A. Bakery; awad_bakery@yahoo.com

Received 2 June 2013; Accepted 22 September 2013

Academic Editor: Abdelghani Bellouquid

Copyright (C) 2013 Awad A. Bakery. This is an open access article distributed under the Creative Commons Attribution License, which permits unrestricted use, distribution, and reproduction in any medium, provided the original work is properly cited.

We introduced the ideal convergence of generalized difference sequence spaces combining an infinite matrix of complex numbers with respect to $\lambda$-sequences and the Musielak-Orlicz function over $n$-normed spaces. We also studied some topological properties and inclusion relations between these spaces.

\section{Introduction}

Throughout the paper $\omega, \ell_{\infty}, c, c_{0}$, and $\ell_{p}$ denote the classes of all, bounded, convergent, null, and p-absolutely summable sequences of complex numbers. The sets of natural numbers and real numbers will be denoted by $\mathbb{N}$ and $\mathbb{R}$, respectively. Many authors studied various sequence spaces using normed or seminormed linear spaces. In this paper, using an infinite matrix of complex numbers and the notion of ideal, we aimed to introduce some new sequence spaces with respect to generalized difference operator $\Delta_{m}^{s}$ on $\lambda$-sequences and the Musielak-Orlicz function in $n$-normed linear spaces. By an ideal we mean a family $I \subset 2^{Y}$ of subsets of a nonempty set $Y$ satisfying the following: (i) $\phi \in I$; (ii) $A, B \in$ $I$ imply $A \cup B \in I$; (iii) $A \in I, B \subset A$ imply $B \in I$, while an admissible ideal $I$ of $Y$ further satisfies $\{x\} \in I$ for each $x \in Y$. The notion of ideal convergence was introduced first by Kostyrko et al. [1] as a generalization of statistical convergence. The concept of 2-normed spaces was initially introduced by Gähler [2] in the 1960s, while that of $n$-normed spaces can be found in [3]; this concept has been studied by many authors; see for instance [4-7]. The notion of ideal convergence in a 2-normed space was initially introduced by Gürdal [8]. Later on, it was extended to $n$-normed spaces by Gürdal and Şahiner [9]. Given that $I \subset 2^{\mathbb{N}}$ is a nontrivial ideal in $\mathbb{N}$, the sequence $\left(x_{n}\right)_{n \in \mathbb{N}}$ in a normed space $(X ;\|\cdot\|)$ is said to be $I$-convergent to $x \in X$, if, for each $\varepsilon>0$,

$$
A(\varepsilon)=\left\{n \in \mathbb{N}:\left\|x_{n}-x\right\| \geq \varepsilon\right\} \in I .
$$

A sequence $\left(x_{k}\right)$ in a normed space $(X,\|\cdot\|)$ is said to be $I$-bounded if there exists $L>0$ such that

$$
\left\{k \in \mathbb{N}:\left\|x_{k}\right\|>L\right\} \in I .
$$

A sequence $\left(x_{k}\right)$ in a normed space $(X,\|\cdot\|)$ is said to be $I$-Cauchy if, for each $\varepsilon>0$, there exists a positive integer $m=m(\varepsilon)$ such that

$$
\left\{k \in \mathbb{N}:\left\|x_{k}-x_{m}\right\| \geq \varepsilon\right\} \in I \text {. }
$$

In paper [10], the notion of $\lambda$-convergent and bounded sequences is introduced as follows: let $\lambda=\left(\lambda_{j}\right)_{j=1}^{\infty}$ be a strictly increasing sequence of positive real numbers tending to infinity; that is,

$$
0<\lambda_{1}<\lambda_{2}<\cdots, \quad \lambda_{j} \longrightarrow \infty \quad \text { as } j \longrightarrow \infty .
$$

We say that a sequence $x=\left(x_{j}\right) \in \omega$ is $\lambda$-convergent to the number $l \in \mathbb{C}$, called the $\lambda$-limit of $x$, if $\Lambda_{j}(x) \rightarrow$ $l$ as $j \rightarrow \infty$, where

$$
\Lambda_{j}(x)=\frac{1}{\lambda_{j}} \sum_{r=1}^{j}\left(\lambda_{r}-\lambda_{r-1}\right) x_{r}, \quad j \in \mathbb{N} .
$$


The class of all sequences $\left(\lambda_{j}\right)$ satisfying this property is denoted by $\Lambda$.

In particular, we say that $x$ is a $\lambda$-null sequence if $\Lambda_{j}(x) \rightarrow 0$ as $j \rightarrow \infty$. Further, we say that $x$ is $\lambda$ bounded if $\sup _{j}\left|\Lambda_{j}(x)\right|<\infty$. Here and in the sequel, we will use the convention that any term with a zero subscript is equal to naught; for example, $\lambda_{0}=0$ and $x_{0}=0$. Now, it is well known [10] that if $\lim _{j} x_{j}=a$ in the ordinary sense of convergence, then

$$
\lim _{j \rightarrow \infty}\left(\frac{1}{\lambda_{j}} \sum_{r=1}^{j}\left(\lambda_{r}-\lambda_{r-1}\right)\left|x_{r}-a\right|\right)=0 .
$$

This implies that

$$
\begin{aligned}
\lim _{j}\left|\Lambda_{j}(x)-a\right| \\
=\lim _{j \rightarrow \infty}\left|\frac{1}{\lambda_{j}} \sum_{r=1}^{j}\left(\lambda_{r}-\lambda_{r-1}\right)\left(x_{r}-a\right)\right|=0,
\end{aligned}
$$

which yields that $\lim _{j} \Lambda_{j}(x)=a$ and hence $x$ is $\lambda$-convergent to $a$. We therefore deduce that the ordinary convergence implies the $\lambda$-convergence to the same limit.

An Orlicz function is a function $M:[0, \infty) \rightarrow$ $[0, \infty)$ which is continuous, nondecreasing, and convex with $M(0)=0$ and $M(x)>0$ for $x>0$ and $M(x) \rightarrow$ $\infty$, as $x \rightarrow \infty$. If convexity of $M$ is replaced by $M(x+$ $y) \leq M(x)+M(y)$, then it is called a modulus function, introduced by Nakano [11]. Ruckle [12] and Maddox [13] used the idea of a modulus function to construct some spaces of complex sequences. An Orlicz function $M$ is said to satisfy the $\Delta_{2}$-condition for all values of $x \geq 0$, if there exists a constant $k>0$, such that $M(2 x) \leq k M(x)$. The $\Delta_{2^{-}}$ condition is equivalent to $M(l x) \leq k l M(x)$ for all values of $x$ and for $l>1$. Lindentrauss and Tzafriri [14] used the idea of an Orlicz function to define the following sequence spaces:

$$
\ell_{M}=\left\{x \in \omega: \sum_{k=1}^{\infty} M\left(\frac{|x(k)|}{\rho}\right)<\infty\right\},
$$

which is a Banach space with the Luxemburg norm defined by

$$
\|x\|=\inf \left\{\rho>0: \sum_{k=1}^{\infty} M\left(\frac{|x(k)|}{\rho}\right) \leq 1\right\} .
$$

The space $\ell_{M}$ is closely related to the space $\ell_{p}$, which is an Orlicz sequence space with $M(x)=x^{p}$ for $1 \leq p<\infty$. Recently different classes of sequences have been introduced using Orlicz functions. See [7, 9, 15-17].

A sequence $M=\left(M_{k}\right)$ of Orlicz functions $M_{k}$ for all $k \in \mathbb{N}$ is called a Musielak-Orlicz function.

Kizmaz [18] defined the difference sequences $\ell_{\infty}(\Delta), c(\Delta)$, and $c_{0}(\Delta)$ as follows.

$Z(\Delta)=\left\{x=\left(x_{k}\right):\left(\Delta x_{k}\right) \in Z\right\}$. For $Z=\ell_{\infty}, c$, and $c_{0}$, where $\Delta x=\left(x_{k}-x_{k+1}\right)$, for all $k \in \mathbb{N}$. The above spaces are Banach spaces, normed by $\|x\|=\left|x_{1}\right|+\sup _{k}\left|\Delta x_{k}\right|$. The notion of difference sequence spaces was generalized by Et and Colak [19] as follows: $Z\left(\Delta^{s}\right)=\left\{x=\left(x_{k}\right):\left(\Delta^{s} x_{k}\right) \in Z\right\}$. For $Z=\ell_{\infty}, c$ and $c_{0}$, where $s \in \mathbb{N},\left(\Delta^{s} x_{k}\right)=\left(\Delta^{s-1} x_{k}-\right.$ $\left.\Delta^{s-1} x_{k+1}\right)$ and so that $\Delta^{s} x_{k}=\sum_{n=0}^{s}(-1)^{n} C_{n}^{s} x_{k+n}$. Tripathy and Esi [20] introduced the following new type of difference sequence spaces.

$Z\left(\Delta_{m}\right)=\left\{x=\left(x_{k}\right):\left(\Delta_{m} x_{k}\right) \in Z\right\}, Z=\ell_{\infty}, c$, and $c_{0}$, where $\Delta_{m} x_{k}=\left(x_{k}-x_{k+m}\right)$, for all $k \in \mathbb{N}$. Tripathy et al. [21], generalized the previous notions and unified them as follows.

Let $m$ and $s$ be nonnegative integers, then for $Z$ a given sequence space we have

$$
Z\left(\Delta_{m}^{s}\right)=\left\{x=\left(x_{k}\right):\left(\Delta_{m}^{s} x_{k}\right) \in Z\right\}, \text { where }
$$

$$
\begin{gathered}
\Delta_{m}^{s} x_{k}=\sum_{n=0}^{s}(-1)^{n} C_{n}^{s} x_{k+m n} \quad \text { (forward difference), } \\
Z\left(\Delta_{m}^{(s)}\right)=\left\{x=\left(x_{k}\right):\left(\Delta_{m}^{(s)} x_{k}\right) \in Z\right\}, \text { where } \\
\Delta_{m}^{(s)} x_{k}=\sum_{n=0}^{s}(-1)^{n} C_{n}^{s} x_{k-m n} \quad \text { (backward difference), }
\end{gathered}
$$

where $x_{k}=0$, for $k<0$.

\section{Definitions and Preliminaries}

Let $n \in \mathbb{N}$ and $X$ be a linear space over the field $K$ of dimension $d$, where $d \geq n \geq 2$ and $K$ is the field of real or complex numbers. A real valued function $\|\cdot \ldots \cdot\|$ on $X^{n}$ satisfies the following four conditions:

(1) $\left\|x_{1}, x_{2}, \ldots, x_{n}\right\|=0$ if and only if $x_{1}, x_{2}, \ldots$, and $x_{n}$ are linearly dependent in $X$;

(2) $\left\|x_{1}, x_{2}, \ldots, x_{n}\right\|$ is invariant under permutation;

(3) $\left\|\alpha x_{1}, x_{2}, \ldots, x_{n}\right\|=|\alpha|\left\|x_{1}, x_{2}, \ldots, x_{n}\right\|$ for any $\alpha \in K$;

(4) $\left\|x+x^{\prime}, x_{2}, \ldots, x_{n}\right\| \leq\left\|x, x_{2}, \ldots, x_{n}\right\|+\left\|x_{1}, x_{2}, \ldots, x_{n}\right\|$, which is called an $n$-norm on $X$ and the pair $(X ;\|\cdot \ldots \cdot\|)$ is called an $n$-normed space over the field $K$. For example, we may take $X=\mathbb{R}^{n}$ being equipped with the $n$-norm $\left\|x_{1}, x_{2}, \ldots, x_{n}\right\|_{E}=$ the volume of the $n$-dimensional parallelepiped spanned by the vectors $x_{1}, x_{2}, \ldots$, and $x_{n}$ which may be given explicitly by the formula

$$
\begin{aligned}
\left\|x_{1}, x_{2}, \ldots, x_{n}\right\|_{E} & =\left|\operatorname{det}\left(x_{i j}\right)\right| \\
& =\operatorname{abs}\left(\left|\begin{array}{cccc}
x_{11} & x_{12} & \cdots & x_{1 n} \\
x_{21} & x_{22} & \cdots & x_{2 n} \\
\vdots & \vdots & \ddots & \vdots \\
x_{n 1} & x_{n 2} & \cdots & x_{n n}
\end{array}\right|\right),
\end{aligned}
$$

where $x_{i}=\left(x_{i 1}, x_{i 2}, \ldots, x_{i n}\right)$ for each $i \in \mathbb{N}$.

Let $(X,\|\cdot \ldots \cdot\|)$ be an $n$-normed space of dimension $d \geq$ $n \geq 2$ and $\left\{a_{1}, a_{2}, a_{3}, \ldots, a_{n}\right\}$ a linearly independent set in $X$. Then, the function $\|\cdot \ldots \cdot\|_{\infty}$ on $X^{n-1}$ defined by

$$
\left\|x_{1}, x_{2}, \ldots, x_{n}\right\|_{\infty}=\max _{1 \leq i \leq n}\left\|x_{1}, x_{2}, \ldots, x_{n-1}, a_{i}\right\|
$$


defines an $(n-1)$-norm on $X$ with respect to $a_{1}, a_{2}, a_{3}, \ldots$, and $a_{n}$ and this is known as the derived $(n-1)$-norm. The standard $(n)$-norm on $X$, a real inner product space of dimension $d \geq n$, is as follows:

$$
\begin{aligned}
& \left\|x_{1}, x_{2}, \ldots, x_{n}\right\|_{s} \\
& =\operatorname{abs}\left(\left(\begin{array}{cccc}
\left\langle x_{1}, x_{1}\right\rangle & \left\langle x_{1}, x_{2}\right\rangle & \cdots & \left\langle x_{1}, x_{n}\right\rangle \\
\left\langle x_{2}, x_{1}\right\rangle & \left\langle x_{2}, x_{2}\right\rangle & \cdots & \left\langle x_{2}, x_{n}\right\rangle \\
\vdots & \vdots & \ddots & \vdots \\
\left\langle x_{n}, x_{1}\right\rangle & \left\langle x_{n}, x_{2}\right\rangle & \cdots & \left\langle x_{n}, x_{n}\right\rangle
\end{array} \mid\right)^{1 / 2},\right.
\end{aligned}
$$

where $\langle\cdot, \cdot\rangle$ denotes the inner product on $X$. If we take $X=$ $\mathbb{R}^{n}$, then

$$
\left\|x_{1}, x_{2}, \ldots, x_{n}\right\|_{E}=\left\|x_{1}, x_{2}, \ldots, x_{n}\right\|_{s} .
$$

For $n=1$, this $n$-norm is the usual norm $\left\|x_{1}\right\|=$ $\sqrt{\left\langle x_{1}, x_{1}\right\rangle}$.

Definition 1. A sequence $\left(x_{k}\right)$ in an $n$-normed space is said to be convergent to $x \in X$ if

$$
\begin{array}{r}
\lim _{k \rightarrow \infty}\left\|\left(z_{1}, z_{2}, \ldots, z_{n-1}, x_{k}-x\right)\right\|_{n}=0, \\
\forall z_{1}, z_{2}, \ldots, z_{n-1} \in X .
\end{array}
$$

Definition 2. A sequence $\left(x_{k}\right)$ in an $n$-normed space is called Cauchy (with respect to $n$-norm) if

$$
\begin{array}{r}
\lim _{k, j \rightarrow \infty}\left\|z_{1}, z_{2}, \ldots, z_{n-1}, x_{k}-x_{j}\right\|_{n}=0, \\
\forall z_{1}, z_{2}, \ldots, z_{n-1} \in X .
\end{array}
$$

If every Cauchy sequence in $X$ converges to an $x \in X$, then $X$ is said to be complete (with respect to the $n$-norm). A complete $n$-normed space is called an $n$-Banach space.

Definition 3. A sequence $\left(x_{k}\right)$ in an $n$-normed space $(X, \|$. $\ldots \cdot \|)$ is said to be $I$-convergent to $x_{0} \in X$ with respect to $n$ norm, if, for each $\varepsilon>0$, the set

$$
\begin{aligned}
\{k \in \mathbb{N}: & \left\|x_{k}-x_{0}, z_{1}, z_{2}, \ldots, z_{n-1}\right\| \\
& \left.\geq \varepsilon \text {, for every } z_{1}, z_{2}, \ldots, z_{n-1}\right\} \in I .
\end{aligned}
$$

Definition 4. A sequence $\left(x_{k}\right)$ in an $n$-normed space $(X, \|$. $\ldots \cdot \|)$ is said to be $I$-Cauchy if, for each $\varepsilon>0$, there exists a positive integer $m=m(\varepsilon)$ such that the set

$$
\begin{aligned}
\left\{k \in \mathbb{N}:\left\|x_{k}-x_{m}, z_{1}, z_{2}, \ldots, z_{n-1}\right\|\right. \\
\\
\left.\quad \geq \varepsilon \text {, for every } z_{1}, z_{2}, \ldots, z_{n-1}\right\} \in I .
\end{aligned}
$$

Let $x=\left(x_{k}\right)$ be a sequence; then $S(x)$ denotes the set of all permutations of the elements of $\left(x_{k}\right)$; that is, $S(x)=$ $\left(x_{\pi(n)}\right): \pi$ is a permutation of $\mathbb{N}$.

Definition 5. A sequence space $E$ is said to be symmetric if $S(x) \subset E$ for all $x \in E$.
Definition 6. A sequence space $E$ is said to be normal (or solid) if $\left(\alpha_{k} x_{k}\right) \in E$, whenever $\left(x_{k}\right) \in E$ and for all sequences $\left(\alpha_{k}\right)$ of scalars with $\left|\alpha_{k}\right| \leq 1$ for all $k \in \mathbb{N}$.

Definition 7. A sequence space $E$ is said to be a sequence algebra if $x, y \in E$; then $x \cdot y=\left(x_{k} y_{k}\right) \in E$.

Lemma 8. Every n-normed space is an $(n-r)$-normed space for all $r=1,2,3, \ldots, n-1$. In particular, every $n$-normed space is a normed space.

Lemma 9. On a standard n-normed space $X$, the derived ( $n-$ 1)-norm $\|\cdot \ldots \cdot\|_{\infty}$ defined with respect to the orthogonal set $\left\{e_{1}, e_{2}, \ldots, e_{n}\right\}$ is equivalent to the standard $(n-1)$ norm $\|\cdot \ldots \cdot\|_{s}$. To be precise, one has

$$
\left\|x_{1}, x_{2}, \ldots, x_{n-1}\right\|_{\infty} \leq\|\cdot \ldots \cdot\|_{s} \leq \sqrt{n}\left\|x_{1}, x_{2}, \ldots, x_{n-1}\right\|_{\infty},
$$

for all $x_{1}, x_{2}, \ldots, x_{n-1} \in X$, where $\left\|x_{1}, x_{2}, \ldots, x_{n-1}\right\|_{\infty}=$ $\max _{1 \leq i \leq n}\left\{\left\|x_{1}, x_{2}, \ldots, x_{n-1}, e_{i}\right\|_{S}\right\}$.

For any bounded sequence $\left(p_{n}\right)$ of positive numbers, one has the following well known inequality: if $0 \leq p_{k} \leq \sup _{k} p_{k}=$ $G$ and $D=\max \left(1,2^{G-1}\right)$, then $\left|a_{n}+b_{n}\right|^{p_{n}} \leq D\left(\left|a_{n}\right|^{p_{n}}+\left|b_{n}\right|^{p_{n}}\right)$, for all $k$ and $a_{k}, b_{k} \in \mathbb{C}$.

\section{Main Results}

In this section, we define some new ideal convergent sequence spaces and investigate their linear topological structures. We find out some relations related to these sequence spaces. Let $I$ be an admissible ideal of $\mathbb{N}$, $\mathscr{M}=\left(M_{j}\right)$ a Musielak-Orlicz function, and $\Delta_{m}^{s}$ the forward generalized difference operator on the class of all sequences $\left(\lambda_{j}\right)$ satisfying the property $\Lambda$ and an $n$-normed space $(X,\|\cdot \ldots \cdot\|)$. Further, let $p=\left(p_{k}\right)$ be any bounded sequence of positive real numbers; we will define the following sequence spaces:

$$
\begin{aligned}
& W\left[A, \mathscr{M}, \Delta_{m}^{s}, \Lambda, p,\|\cdot \ldots \cdot\|\right]^{I} \\
& =\left\{\begin{array}{c}
x \in \omega(n-X): \forall \varepsilon>0 \\
\quad \times\left\{k \in \mathbb{N}: \sum_{j=1}^{\infty} a_{k j}\right. \\
\times\left[M _ { j } \left(\| \frac{\Delta_{m}^{s}\left(\Lambda_{j}(x)\right)-l}{\rho},\right.\right. \\
\left.\left.\left.z_{1}, z_{2}, \ldots, z_{n-1} \|\right)\right]^{p_{j}} \geq \varepsilon\right\} \in I,
\end{array}\right.
\end{aligned}
$$


for some $\rho>0, l \in X$ and each $\left.z_{1}, z_{2}, \ldots, z_{n-1} \in X\right\}$,

$$
\begin{aligned}
& W\left[A, \mathscr{M}, \Delta_{m}^{s}, \Lambda, p,\|\cdot \ldots \cdot\|\right]_{0}^{I} \\
& =\{x \in \omega(n-X): \forall \varepsilon>0 \\
& \quad \times\left\{k \in \mathbb{N}: \sum_{j=1}^{\infty} a_{k j}\right. \\
& \times\left[M _ { j } \left(\| \frac{\Delta_{m}^{s}\left(\Lambda_{j}(x)\right)}{\rho},\right.\right. \\
& \left.\left.\left.z_{1}, z_{2}, \ldots, z_{n-1} \|\right)\right]^{p_{j}} \geq \varepsilon\right\} \in I,
\end{aligned}
$$

for some $\rho>0$, and each $\left.z_{1}, z_{2}, \ldots, z_{n-1} \in X\right\}$,

$$
\begin{aligned}
& W\left[A, \mathscr{M}, \Delta_{m}^{s}, \Lambda, p,\|\cdot \ldots \cdot\|\right]_{\infty} \\
& =\left\{\begin{array}{l}
x \in \omega(n-X):
\end{array}\right.
\end{aligned}
$$$$
\exists K>0 \text { st. } \sup _{k} \sum_{j=1}^{\infty} a_{k j}
$$$$
\begin{gathered}
\times\left[M _ { j } \left(\| \frac{\Delta_{m}^{s}\left(\Lambda_{j}(x)\right)}{\rho},\right.\right. \\
\left.\left.z_{1}, z_{2}, \ldots, z_{n-1} \|\right)\right]^{p_{j}}
\end{gathered}
$$

$<\infty$

for some $\rho>0$ and each $\left.z_{1}, z_{2}, \ldots, z_{n-1} \in X\right\}$,

$$
\begin{aligned}
& W\left[A, \mathscr{M}, \Delta_{m}^{s}, \Lambda, p,\|\cdot \ldots \cdot\|\right]_{\infty}^{I} \\
& =\left\{\begin{array}{l}
x \in \omega(n-X): \\
\quad \exists K>0, \text { s.t. }\left\{k \in \mathbb{N}: \sum_{j=1}^{\infty} a_{k j}\right. \\
\times\left[M _ { j } \left(\| \frac{\Delta_{m}^{s}\left(\Lambda_{j}(x)\right)}{\rho},\right.\right.
\end{array}\right.
\end{aligned}
$$

$$
\left.\left.\left.z_{1}, z_{2}, \ldots, z_{n-1} \|\right)\right]^{p_{j}} \geq K\right\} \in I
$$

for some $\rho>0$,

$$
\text { and each } \left.z_{1}, z_{2}, \ldots, z_{n-1} \in X\right\} \text {. }
$$

Let us consider a few special cases of the aforementioned sets.

(1) If $M_{k}(x)=M(x)$, for all $k \in \mathbb{N}$ then the previous classes of sequences are denoted by $W\left[A, M, \Delta_{m}^{s}, \Lambda, p, \| \cdot \ldots\right.$. $\|]^{I}, W\left[A, M, \Delta_{m}^{s}, \Lambda, p,\|\cdot \ldots \cdot\|\right]_{0}^{I}, W\left[A, M, \Delta_{m}^{s}, \Lambda, p,\|\cdot \ldots \cdot\|\right]_{\infty}$, and $W\left[A, M, \Delta_{m}^{s}, \Lambda, p,\|\cdot \ldots \cdot\|\right]_{\infty}^{I}$, respectively.

(2) If $p_{k}=1$ for all $k \in \mathbb{N}$ then the previous classes of sequences are denoted by $W\left[A, \mathscr{M}, \Delta_{m}^{s}, \Lambda,\|\cdot \ldots \cdot\|\right]^{I}$, $W\left[A, \mathscr{M}, \Delta_{m}^{s}, \Lambda,\|\cdot \ldots \cdot\|\right]_{0}^{I}, W\left[A, \mathscr{M}, \Delta_{m}^{s}, \Lambda,\|\cdot \ldots \cdot\|\right]_{\infty}$, and $W\left[A, \mathscr{M}, \Delta_{m}^{s}, \Lambda,\|\cdot \ldots \cdot\|\right]_{\infty}^{I}$, respectively.

(3) If $M_{k}(x)=x$, for all $k \in \mathbb{N}$ and $x \in[0, \infty[$, then the previous classes of sequences are denoted by $W\left[A, \Delta_{m}^{s}, \Lambda, p,\|\cdot \ldots \cdot\|\right]^{I}, W\left[A, \Delta_{m}^{s}, \Lambda, p,\|\cdot \ldots \cdot\|\right]_{0}^{I}$, $W\left[A, \Delta_{m}^{s}, \Lambda, p,\|\cdot \ldots \cdot\|\right]_{\infty}$, and $W\left[A, \Delta_{m}^{s}, \Lambda, p,\|\cdot \ldots \cdot\|\right]_{\infty}^{I}$, respectively.

(4) If we take $M_{k}(x)=M(x)$, for all $k \in \mathbb{N a n d} A=$ $\left(a_{k j}\right)$ as

$$
a_{k j}= \begin{cases}\frac{1}{k}, & k \geq j \\ 0, & \text { otherwise }\end{cases}
$$

then we denote the previous classes of sequences by $W\left[C, M, \Delta_{m}^{s}, \Lambda, p,\|\cdot \ldots \cdot\|\right]^{I}, W\left[C, M, \Delta_{m}^{s}, \Lambda, p,\|\cdot \ldots \cdot\|\right]_{0}^{I}$, $W\left[C, M, \Delta_{m}^{s}, p,\|\cdot \ldots \cdot\|\right]_{\infty}$, and $W\left[C, M, \Delta_{m}^{s}, \Lambda, p,\|\cdot \ldots \cdot\|\right]_{\infty}^{I}$, respectively.

(5) If we take $M_{k}(x)=M(x)$ and $A=\left(a_{k j}\right)$ as

$$
a_{k j}= \begin{cases}\frac{1}{\phi_{k}}, & j \in I_{k}=\left[k-\phi_{k}+1, k\right] \\ 0, & \text { otherwise }\end{cases}
$$

where $\left(\phi_{k}\right)$ is a nondecreasing sequence of positive numbers tending to $\infty, \phi_{1}=1$, and $\phi_{k+1} \leq \phi_{k}+1$, then we denote the previous classes of sequences by $W\left[\Phi, M, \Delta_{m}^{s}, \Lambda, p, \| \cdot \ldots\right.$. $\|]^{I}, W\left[\Phi, M, \Delta_{m}^{s}, \Lambda, p,\|\cdot \ldots \cdot\|\right]_{0}^{I}, W\left[\Phi, M, \Delta_{m}^{s}, \Lambda, p,\|\cdot \ldots \cdot\|\right]_{\infty}$, and $W\left[\Phi, M, \Delta_{m}^{s}, \Lambda, p,\|\cdot \ldots \cdot\|\right]_{\infty}^{I}$.

(6) If $A=\left(a_{k j}\right)$ as in (22), then we denote the previous classes of sequences by $W\left[\Phi, \mathscr{M}, \Delta_{m}^{s}, \Lambda, p,\|\cdot \ldots \cdot\|\right]^{I}$, $W\left[\Phi, \mathscr{M}, \Delta_{m}^{s}, \Lambda, p,\|\cdot \ldots \cdot\|\right]_{0}^{I}, W\left[\Phi, \mathscr{M}, \Delta_{m}^{s}, \Lambda, p,\|\cdot \ldots \cdot\|\right]_{\infty}$, and $W\left[\Phi, \mathscr{M}, \Delta_{m}^{s}, \Lambda, p,\|\cdot \ldots \cdot\|\right]_{\infty}^{I}$.

And if $\lambda_{j}=j$ for all $j \in \mathbb{N}$, then the previous classes of sequences are denoted by $W\left[\Phi, \mathscr{M}, \Delta_{m}^{s}, C, p,\|\cdot \ldots \cdot\|\right]^{I}$, $W\left[\Phi, \mathscr{M}, \Delta_{m}^{s}, C, p,\|\cdot \ldots \cdot\|\right]_{0}^{I}, W\left[\Phi, \mathscr{M}, \Delta_{m}^{s}, C, p, \| \cdot \ldots \cdot\right.$ $\|]_{\infty}$, and $W\left[\Phi, \mathscr{M}, \Delta_{m}^{s}, C, p,\|\cdot \ldots \cdot\|\right]_{\infty}^{I}$ and they are a 
generalization of the sequence spaces defined by Bakery et al. [22].

(7) By a lacunary $\theta=\left(j_{r}\right), r=0,1,2, \ldots$, where $j_{0}=0$, we will mean an increasing sequence of nonnegative integers with $j_{r}-j_{r-1} \rightarrow \infty$ as $r \rightarrow \infty$. The interval determined by $\theta$ will be denoted by $\left.\left.I_{r}=\right] j_{r-1}, j_{r}\right]$ and $h_{r}=j_{r}-j_{r-1}$ and let $A=\left(a_{k j}\right)$ as

$$
a_{k j}= \begin{cases}\frac{1}{h_{r}}, & \left.\left.j \in I_{r}=\right] j_{r-1}, j_{r}\right] \\ 0, & \text { otherwise. }\end{cases}
$$

Then we denote the previous classes of sequences by $W\left[\theta, M, \Delta_{m}^{s}, \Lambda, p,\|\cdot \ldots \cdot\|\right]^{I}, W\left[\theta, M, \Delta_{m}^{s}, \Lambda, p,\|\cdot \ldots \cdot\|\right]_{0}^{I}$, $W\left[\theta, M, \Delta_{m}^{s}, \Lambda, p,\|\cdot \ldots \cdot\|\right]_{\infty}$, and $W\left[\theta, M, \Delta_{m}^{s}, \Lambda, p, \| \cdot \ldots \cdot\right.$ $\|]_{\infty}^{I}$, respectively.

(8) If $M_{k}(x)=M(x)$, for all $k \in \mathbb{N}, A=I$, and $\lambda_{j}=j$, then the previous classes of sequences are denoted by $W\left[M, \Delta_{m}^{s}, C, p,\|\cdot \ldots \cdot\|\right]^{I}, W\left[M, \Delta_{m}^{s}, C, p, \| \cdot \ldots \cdot\right.$ $\|]_{0}^{I}, W\left[M, \Delta_{m}^{s}, C, p,\|\cdot \ldots \cdot\|\right]_{\infty}$, and $W\left[M, \Delta_{m}^{s}, C, p,\|\cdot \ldots \cdot\|\right]_{\infty}^{I}$.

(9) If $s=1$, then the previous classes of sequences are denoted by $W\left[A, \mathscr{M}, \Delta_{m}, \Lambda, p,\|\cdot \ldots \cdot\|\right]^{I}, W\left[M, \Delta_{m}, C, p, \| \cdot\right.$ $\ldots \cdot \|]_{0}^{I}, W\left[M, \Delta_{m}, C, p,\|\cdot \ldots \cdot\|\right]_{\infty}$, and $W\left[M, \Delta_{m}, C, p, \| \cdot\right.$ $\ldots \cdot \|]_{\infty}^{I}$.

(10) If $m=1$, then the previous classes of sequences are denoted by $\left[A, \mathscr{M}, \Delta^{s}, \Lambda, p,\|\cdot \ldots \cdot\|\right]^{I}, W\left[M, \Delta^{s}, C, p,\|\cdot \ldots \cdot\|\right]_{0}^{I}$, $W\left[M, \Delta^{s}, C, p,\|\cdot \ldots \cdot\|\right]_{\infty}$, and $W\left[M, \Delta^{s}, C, p,\|\cdot \ldots \cdot\|\right]_{\infty}^{I}$.

Theorem 10. The spaces $W\left[A, \mathscr{M}, \Delta_{m}^{s}, \Lambda, p,\|\cdot \ldots \cdot\|\right]^{I}$, $W\left[A, \mathscr{M}, \Delta_{m}^{s}, \Lambda, p,\|\cdot \ldots \cdot\|\right]_{0}^{I}$ and $W\left[A, \mathscr{M}, \Delta_{m}^{s}, \Lambda, p,\|\cdot \ldots \cdot\|\right]_{\infty}^{I}$ are linear spaces.

Proof. We will prove the assertion for $W\left[A, \mathscr{M}, \Delta_{m}^{s}, \Lambda, p, \| \cdot\right.$ $\ldots \cdot \|]_{0}^{I}$; the others can be proved similarly. Assume that $x=$ $\left(x_{k}\right), y=\left(y_{k}\right) \in W\left[A, \mathscr{M}, \Delta_{m}^{s}, \Lambda, p,\|\cdot \ldots \cdot\|\right]_{0}^{I}$, and $\alpha, \beta \in \mathbb{C}$. Then, there exist $\rho_{1}$ and $\rho_{2}$ such that the sets

$$
\begin{aligned}
& \left\{k \in \mathbb{N}: \sum_{j=1}^{\infty} a_{k j}\right. \\
& \quad \times\left[M _ { j } \left(\| \frac{\Delta_{m}^{s}\left(\Lambda_{j}(x)\right)}{\rho_{1}},\right.\right. \\
& \left.\geq \frac{\varepsilon}{2}\right\} \in I, \\
& \left\{\begin{array}{l}
\left.\left.z_{1}, z_{2}, \ldots, z_{n-1} \|\right)\right]^{p_{j}} \\
k \in \mathbb{N}:
\end{array}\right.
\end{aligned}
$$

$$
\begin{aligned}
& \sum_{j=1}^{\infty} a_{k j}\left[M_{j}\left(\left\|\frac{\Delta_{m}^{s}\left(\Lambda_{j}(y)\right.}{\rho_{2}}, z_{1}, z_{2}, \ldots, z_{n-1}\right\|\right)\right]^{p_{j}} \\
& \left.\geq \frac{\varepsilon}{2}\right\} \in I .
\end{aligned}
$$

Since $(X,\|\cdot \ldots \cdot\|)$ is an $n$-norm, $\Delta_{m}^{s}$ and $\Lambda_{j}$ are linear, and the Orlicz function $M_{j}$ is convex for all $j \in \mathbb{N}$, the following inequality holds:

$$
\begin{aligned}
& \sum_{j=1}^{\infty} a_{k j}\left[M _ { j } \left(\| \frac{\Delta_{m}^{s}\left(\Lambda_{j}(\alpha x+\beta y)\right)}{|\alpha| \rho_{1 F}+|\beta| \rho_{2}},\right.\right. \\
& \left.\left.z_{1}, z_{2}, \ldots, z_{n-1} \|\right)\right]^{p_{j}} \\
& \leq D \sum_{j=1}^{\infty} a_{k j} \frac{|\alpha| \rho_{1}}{|\alpha| \rho_{1}+|\beta| \rho_{2}} \\
& \times\left[M_{j}\left(\left\|\frac{\Delta_{m}^{s}\left(\Lambda_{j}(x)\right)}{\rho_{1}}, z_{1}, z_{2}, \ldots, z_{n-1}\right\|\right)\right]^{p_{j}} \\
& +D \sum_{j=1}^{\infty} a_{k j} \frac{|\beta| \rho_{2}}{|\alpha| \rho_{1}+|\beta| \rho_{2}} \\
& \times\left[M_{j}\left(\left\|\frac{\Delta_{m}^{s}\left(\Lambda_{j}(y)\right)}{\rho_{2}}, z_{1}, z_{2}, \ldots, z_{n-1}\right\|\right)\right]^{p_{j}} \\
& \leq D L \sum_{j=1}^{\infty} a_{k j} \\
& \times\left[M_{j}\left(\left\|\frac{\Delta_{m}^{s}\left(\Lambda_{j}(x)\right)}{\rho_{1}}, z_{1}, z_{2}, \ldots, z_{n-1}\right\|\right)\right]^{p_{j}} \\
& +D L \sum_{j=1}^{\infty} a_{k j} \\
& \times\left[M _ { j } \left(\| \frac{\Delta_{m}^{s}\left(\Lambda_{j}(y)\right)}{\rho_{2}},\right.\right. \\
& \left.\left.z_{1}, z_{2}, \ldots, z_{n-1} \|\right)\right]^{p_{j}},
\end{aligned}
$$

where $L=\max \left\{|\alpha| \rho_{1} /\left(|\alpha| \rho_{1}+|\beta| \rho_{2}\right),|\beta| \rho_{2} /\left(|\alpha| \rho_{1}+|\beta| \rho_{2}\right)\right\}$. On the other hand from the above inequality we get

$$
\left\{k \in \mathbb{N}: \sum_{j=1}^{\infty} a_{k j}\left[M _ { j } \left(\| \frac{\Delta_{m}^{s}\left(\Lambda_{j}(\alpha x+\beta y)\right)}{|\alpha| \rho_{1}+|\beta| \rho_{2}},\right.\right.\right.
$$




$$
\begin{aligned}
& \left.\left.\left.z_{1}, z_{2}, \ldots, z_{n-1} \|\right)\right]^{p_{j}} \geq \varepsilon\right\} \\
& \subseteq\{k \in \mathbb{N}: D L \\
& \times \sum_{j=1}^{\infty} a_{k j}\left[M _ { j } \left(\| \frac{\Delta_{m}^{s}\left(\Lambda_{j}(x)\right)}{\rho_{1}}\right.\right. \\
& \left.\left.\left.z_{1}, z_{2}, \ldots, z_{n-1} \|\right)\right]^{p_{j}} \geq \frac{\varepsilon}{2}\right\} \\
& \cup\{k \in \mathbb{N}: D L \\
& \times \sum_{j=1}^{\infty} a_{k j}\left[M_{j}\left(\left\|\frac{\Delta_{m}^{s}\left(\Lambda_{j}(y)\right)}{\rho_{2}}, z_{1}, z_{2}, \ldots, z_{n-1}\right\|\right)\right]^{p_{j}} \\
& \left.\geq \frac{\varepsilon}{2}\right\} \text {. }
\end{aligned}
$$

Since the two sets on the right hand side belong to $I$, this completes the proof.

Theorem 11. The spaces $W\left[A, \mathscr{M}, \Delta_{m}^{s}, \Lambda, p,\|\cdot \ldots \cdot\|\right]^{I}$, $W\left[A, \mathscr{M}, \Delta_{m}^{s}, \Lambda, p,\|\cdot \ldots \cdot\|\right]_{0}^{I}$, and $W\left[A, \mathscr{M}, \Delta_{m}^{s}, \Lambda, p,\|\cdot \ldots \cdot\|\right]_{\infty}^{I}$ are paranormed spaces (not totally paranormed) with respect to the paranorm $g_{\Delta}$ defined by

$$
\begin{aligned}
& g_{\Delta}(x) \\
& =\sum_{j=1}^{m s}\left\|x_{j}, z_{1}, z_{2}, \ldots, z_{n-1}\right\| \\
& +\inf \left\{\rho^{p_{k} / H}:\right. \\
& \sup _{k}\left[\sum _ { j = 1 } ^ { \infty } a _ { k j } \left[M _ { j } \left(\| \frac{\Delta_{m}^{s}\left(\Lambda_{j}(x)\right)}{\rho_{1}},\right.\right.\right. \\
& \left.\left.\left.z_{1}, z_{2}, \ldots, z_{n-1} \|\right)\right]^{p_{j}}\right]^{1 / H} \\
& \leq 1, \text { for some } \rho>0 \text {, } \\
& \text { and each } \left.z_{1}, z_{2}, \ldots, z_{n-1} \in X\right\} \text {, }
\end{aligned}
$$

Proof. Clearly $g_{\Delta}(-x)=g_{\Delta}(x)$ and $g_{\Delta}(\theta)=0$. Let $x=$ $\left(x_{k}\right)$ and $y=\left(y_{k}\right) \in W\left[A, \mathscr{M}, \Delta_{m}^{s}, \Lambda, p,\|\cdot \ldots \cdot\|\right]_{0}^{I}$. Then, for $\rho>0$ we set

$$
\begin{aligned}
A_{1}=\left\{\rho: \sup _{k}\right. & {\left[\sum_{j=1}^{\infty} a_{k j}\right.} \\
\times & {\left[M _ { j } \left(\| \frac{\Delta_{m}^{s}\left(\Lambda_{j}(x)\right)}{\rho},\right.\right.} \\
& \left.\left.\left.z_{1}, z_{2}, \ldots, z_{n-1} \|\right)\right]^{p_{j}}\right]^{1 / H} \leq 1,
\end{aligned}
$$$$
\text { for each } \left.z_{1}, z_{2}, \ldots, z_{n-1} \in X\right\} \text {, }
$$

$A_{2}$

$$
=\left\{\rho: \sup _{k}\left[\sum_{j=1}^{\infty} a_{k j}\right.\right.
$$

$$
\times\left[M _ { j } \left(\| \frac{\Delta_{m}^{s}\left(\Lambda_{j}(y)\right)}{\rho},\right.\right.
$$$$
\left.\left.\left.z_{1}, z_{2}, \ldots, z_{n-1} \|\right)\right]^{p_{j}}\right]^{1 / H}
$$$$
\left.\leq 1, \quad \text { for each } z_{1}, z_{2}, \ldots, z_{n-1} \in X\right\} \text {. }
$$

$$
\text { Let } \rho_{1} \in A_{1}, \rho_{2} \in A_{2} \text {, and } \rho=\rho_{1}+\rho_{2} \text {; then we have }
$$

$$
\sup _{k}\left[\sum_{j=1}^{\infty} a_{k j}\right.
$$

$$
\begin{gathered}
\times\left[M _ { j } \left(\| \frac{\Delta_{m}^{s}\left(\Lambda_{j}(x+y)\right)}{\rho},\right.\right. \\
\left.\left.\left.z_{1}, z_{2}, \ldots, z_{n-1} \|\right)\right]^{p_{j}}\right]^{1 / H}
\end{gathered}
$$




$$
\begin{aligned}
& \leq \frac{\rho_{1}}{\rho_{1}+\rho_{2}} \\
& \times \sup _{k}\left[\sum _ { j = 1 } ^ { \infty } a _ { k j } \left[M _ { j } \left(\| \frac{\Delta_{m}^{s}\left(\Lambda_{j}(x)\right)}{\rho_{1}},\right.\right.\right. \\
& \left.\left.\left.z_{1}, z_{2}, \ldots, z_{n-1} \|\right)\right]^{p_{j}}\right]^{1 / H} \\
& +\frac{\rho_{2}}{\rho_{1}+\rho_{2}} \\
& \times \sup _{k}\left[\sum _ { j = 1 } ^ { \infty } a _ { k j } \left[M _ { j } \left(\| \frac{\Delta_{m}^{s}\left(\Lambda_{j}(y)\right)}{\rho_{2}},\right.\right.\right. \\
& \left.\left.\left.z_{1}, z_{2}, \ldots, z_{n-1} \|\right)\right]^{p_{j}}\right]^{1 / H} \leq 1, \\
& g_{\Delta}(x+y) \\
& =\sum_{j=1}^{m s}\left\|x_{j}+y_{j}, z_{1}, z_{2}, \ldots, z_{n-1}\right\| \\
& +\inf \left\{\left(\rho_{1}+\rho_{2}\right)^{p_{k} / H}: \rho_{1} \in A_{1}, \rho_{2} \in A_{2}\right\} \\
& \leq \sum_{j=1}^{m s}\left\|x_{j}, z_{1}, z_{2}, \ldots, z_{n-1}\right\| \\
& +\inf \left\{\left(\rho_{1}\right)^{p_{k} / H}: \rho_{1} \in A_{1}\right\} \\
& +\sum_{j=1}^{m s}\left\|y_{j}, z_{1}, z_{2}, \ldots, z_{n-1}\right\| \\
& +\inf \left\{\left(\rho_{2}\right)^{p_{k} / H}: \rho_{2} \in A_{2}\right\} \\
& =g_{\Delta}(x)+g_{\Delta}(y) \text {. }
\end{aligned}
$$

Let $\lambda^{t} \rightarrow \lambda$ where $\lambda^{t}, \lambda \in \mathbb{C}$, and let $g_{\Delta}\left(x^{t}-x\right) \rightarrow 0$ as $t \rightarrow \infty$. We have to show that $g_{\Delta}\left(\lambda^{t} x^{t}-\lambda x\right) \rightarrow 0$ as $t \rightarrow \infty$. We set

$A_{3}$

$$
\begin{aligned}
& =\left\{\rho_{t}: \sup _{k}\left[\sum_{j=1}^{\infty} a_{k j}\right.\right. \\
& \times\left[M _ { j } \left(\| \frac{\Delta_{m}^{s}\left(\Lambda_{j}(x)\right)}{\rho_{t}},\right.\right.
\end{aligned}
$$

$$
\left.\left.\left.z_{1}, z_{2}, \ldots, z_{n-1} \|\right)\right]^{p_{j}}\right]^{1 / H} \leq 1,
$$$$
\text { for each } \left.z_{1}, z_{2}, \ldots, z_{n-1} \in X\right\} \text {, }
$$$$
\begin{aligned}
A_{4}=\left\{\rho_{t}^{1}: \sup _{k}[\right. & \sum_{j=1}^{\infty} a_{k j} \\
& \times\left[M _ { j } \left(\| \frac{\Delta_{m}^{s}\left(\Lambda_{j}(y)\right)}{\rho_{t}^{1}},\right.\right.
\end{aligned}
$$$$
\left.\left.\left.z_{1}, z_{2}, \ldots, z_{n-1} \|\right)\right]^{p_{j}}\right]^{1 / H} \leq 1,
$$$$
\text { for each } \left.z_{1}, z_{2}, \ldots, z_{n-1} \in X\right\} \text {. }
$$

If $\rho_{t} \in A_{3}$ and $\rho_{t}^{1} \in A_{4}$, then by using non-decreasing and convexity of the Orlicz function $M_{j}$ for all $j \in \mathbb{N}$ we get

$$
\begin{aligned}
\sup _{k}\left[\sum_{j=1}^{\infty} a_{k j}\right. & \\
\times & {\left[M _ { j } \left(\| \frac{\Delta_{m}^{s}\left(\lambda^{t} x_{j}^{t}-\lambda x_{j}\right)}{\left|\lambda^{t}-\lambda\right| \rho_{t}+|\lambda| \rho_{t}^{1}},\right.\right.}
\end{aligned}
$$

$$
\left.\left.\left.z_{1}, z_{2}, \ldots, z_{n-1} \|\right)\right]^{p_{j}}\right]^{1 / H}
$$

$$
\begin{aligned}
\leq \sup _{k}\left[\sum_{j=1}^{\infty} a_{k j}\right. & \\
& \times\left[M _ { j } \left(\| \frac{\left(\Delta_{m}^{s} \lambda^{t} x_{j}^{t}-\lambda x_{j}^{t}\right)}{\left|\lambda^{t}-\lambda\right| \rho_{t}+|\lambda| \rho_{t}^{1}},\right.\right. \\
+\sup _{k}\left[\sum_{j=1}^{\infty} a_{k j}\right. &
\end{aligned}
$$




$$
\begin{aligned}
& \times\left[M _ { j } \left(\| \frac{\Delta_{m}^{s}\left(\lambda x_{j}^{t}-\lambda x_{j}\right)}{\left|\lambda^{t}-\lambda\right| \rho_{t}+|\lambda| \rho_{t}^{1}}\right.\right. \\
& \left.\left.\left.z_{1}, z_{2}, \ldots, z_{n-1} \|\right)\right]^{p_{j}}\right]^{1 / H} \\
& \leq \frac{\left|\lambda^{t}-\lambda\right| \rho_{t}}{\left|\lambda^{t}-\lambda\right| \rho_{t}+|\lambda| \rho_{t}^{1}} \\
& \times \sup _{k}\left[\sum_{j=1}^{\infty} a_{k j}\left[M_{j}\left(\left\|\frac{\Delta_{m}^{s} x_{j}^{t}}{\rho_{t}}, z_{1}, z_{2}, \ldots, z_{n-1}\right\|\right)\right]^{p_{j}}\right]^{1 / H} \\
& +\frac{|\lambda| \rho_{t}^{1}}{\left|\lambda^{t}-\lambda\right| \rho_{t}+|\lambda| \rho_{t}^{1}} \\
& \times \sup _{k}\left[\sum _ { j = 1 } ^ { \infty } a _ { k j } \left[M _ { j } \left(\| \frac{\Delta_{m}^{s}\left(x_{j}^{t}-x_{j}\right)}{\rho_{t}^{1}},\right.\right.\right. \\
& \left.\left.\left.z_{1}, z_{2}, \ldots, z_{n-1} \|\right)\right]^{p_{j}}\right]^{1 / H}
\end{aligned}
$$

From the previous inequality, it follows that

$$
\begin{aligned}
\sup _{k}\left[\sum_{j=1}^{\infty} a_{k j}\right. & \\
\times & {\left[M _ { j } \left(\| \frac{\Delta_{m}^{s}\left(\lambda^{t} x_{j}^{t}-\lambda x_{j}\right)}{\left|\lambda^{t}-\lambda\right| \rho_{t}+|\lambda| \rho_{t}^{1}},\right.\right.} \\
& \left.\left.\left.z_{1}, z_{2}, \ldots, z_{n-1} \|\right)\right]^{p_{j}}\right]^{1 / H} \leq 1,
\end{aligned}
$$

and consequently

$$
\begin{aligned}
& g_{\Delta}\left(\lambda^{t} x^{t}-\lambda x\right) \\
& =\sum_{j=1}^{m s}\left\|\lambda^{t} x_{j}^{t}-\lambda x_{j}, z_{1}, z_{2}, \ldots, z_{n-1}\right\| \\
& \quad+\inf \left\{\left(\left|\lambda^{t}-\lambda\right| \rho_{t}+|\lambda| \rho_{t}^{1}\right)^{p_{k} / H}: \rho_{t} \in A_{3}, \rho_{t}^{1} \in A_{4}\right\}
\end{aligned}
$$

$$
\begin{aligned}
& \leq\left|\lambda^{t}-\lambda\right| \sum_{j=1}^{m s}\left\|x_{j}^{t}, z_{1}, z_{2}, \ldots, z_{n-1}\right\| \\
& \quad+\left|\lambda^{t}-\lambda\right|^{p_{k} / H} \inf \left\{\left(\rho_{t}\right)^{p_{k} / H}: \rho_{t} \in A_{3}\right\} \\
& \quad+|\lambda| \sum_{j=1}^{m s}\left\|\lambda^{t} x_{j}^{t}-\lambda x_{j}, z_{1}, z_{2}, \ldots, z_{n-1}\right\| \\
& \quad+|\lambda|^{p_{k} / H} \inf \left\{\left(\rho_{t}^{1}\right)^{p_{k} / H}: \rho_{t}^{1} \in A_{4}\right\} \\
& \leq \max \left\{\left|\lambda^{t}-\lambda\right|,\left|\lambda^{t}-\lambda\right|^{p_{k} / H}\right\} g_{\Delta}\left(x^{t}\right) \\
& \quad+\max \left\{|\lambda|,|\lambda|^{p_{k} / H}\right\} g_{\Delta}\left(x^{t}-x\right) .
\end{aligned}
$$

Note that $g_{\Delta}\left(x^{t}\right) \leq g_{\Delta}(x)+g_{\Delta}\left(x^{t}-x\right)$, for all $t \in \mathbb{N}$. Hence, by our assumption, the right hand of (34) tends to 0 as $t \rightarrow \infty$, and the result follows. This completes the proof of the theorem.

Theorem 12. Let $\mathscr{M}=\left(M_{j}\right), \mathscr{M}^{\prime}=\left(M_{j}^{\prime}\right)$, and $\mathscr{M}^{\prime \prime}=\left(M_{j}^{\prime \prime}\right)$ be the Musielak-Orlicz functions. Then, the following hold:

(a) $W\left[A, \mathscr{M}^{\prime}, \Delta_{m}^{s}, \Lambda, p,\|\quad \cdot \quad \ldots \quad \cdot \quad\|\right]_{0}^{I} \quad \subseteq$ $W\left[A, \mathscr{M} . \mathscr{M}^{\prime}, \Delta_{m}^{s}, \Lambda, p,\|\cdot \ldots \cdot\|\right]_{0}^{I}$, provided $p=\left(p_{k}\right)$ such that $G_{0}=\inf p_{k}>0$,

(b) $W\left[A, \mathscr{M}^{\prime}, \Delta_{m}^{s}, \Lambda, p,\|\cdot \ldots \cdot\|\right]_{0}^{I} \subseteq W\left[A, \mathscr{M}^{\prime}+\right.$ $\left.\mathscr{M}^{\prime \prime}, \Delta_{m}^{s}, \Lambda, p,\|\cdot \ldots \cdot\|\right]_{0}^{I}$.

Proof. (a) Let $\varepsilon>0$ be given. Choose $\varepsilon_{1}>0$ such that $\sup _{k}\left(\sum_{j=1}^{\infty} a_{k j}\right) \max \left\{\varepsilon_{1}^{G}, \varepsilon_{1}^{G^{0}}\right\}<\varepsilon$. Using the continuity of the Orlicz function $M$, choose $0<\delta<1$ such that $0<t<$ $\delta$ implies that $M(t)<\varepsilon_{1}$.

Let $x=\left(x_{k}\right)$ be any element in $W\left[A, \mathscr{M}^{\prime}, \Delta_{m}^{s}, \Lambda, p, \| \cdot \ldots\right.$. $\|]_{0}^{I}$ and put

$$
A_{\delta}=\{k \in \mathbb{N}:
$$

$$
\begin{aligned}
& \sum_{j=1}^{\infty} a_{k j}\left[M_{j}^{\prime}\left(\left\|\frac{\Delta_{m}^{s}\left(\Lambda_{j}(x)\right)}{\rho_{1}}, z_{1}, z_{2}, \ldots, z_{n-1}\right\|\right)\right]^{p_{j}} \\
& \left.\geq \delta^{G}\right\} .
\end{aligned}
$$

Then, by the definition of ideal convergent, we have the set $A_{\delta} \in I$. If $n \notin A_{\delta}$, then we have 


$$
\begin{aligned}
& \sum_{j=1}^{\infty} a_{k j}\left[M_{j}^{\prime}\left(\left\|\frac{\Delta_{m}^{s}\left(\Lambda_{j}(x)\right)}{\rho_{1}}, z_{1}, z_{2}, \ldots, z_{n-1}\right\|\right)\right]^{p_{j}} \\
& \quad<\delta^{G}\left[M_{j}^{\prime}\left(\left\|\frac{\Delta_{m}^{s}\left(\Lambda_{j}(x)\right)}{\rho_{1}}, z_{1}, z_{2}, \ldots, z_{n-1}\right\|\right)\right]^{p_{j}} \\
& <\delta^{G} \Longrightarrow M_{j}^{\prime}\left(\left\|\frac{\Delta_{m}^{s}\left(\Lambda_{j}(x)\right)}{\rho_{1}}, z_{1}, z_{2}, \ldots, z_{n-1}\right\|\right)<\delta .
\end{aligned}
$$

Using the continuity of the Orlicz function $M_{j}$ for all $j$ and the relation (36), we have

$$
M_{j}\left[M_{j}^{\prime}\left(\left\|\frac{\Delta_{m}^{s}\left(\Lambda_{j}(x)\right)}{\rho_{1}}, z_{1}, z_{2}, \ldots, z_{n-1}\right\|\right)\right]<\varepsilon_{1} .
$$

Consequently, we get

$$
\begin{aligned}
& \sum_{j=1}^{\infty} a_{k j}\left[M_{j} M_{j}^{\prime}\left(\left\|\frac{\Delta_{m}^{s}\left(\Lambda_{j}(x)\right)}{\rho_{1}}, z_{1}, z_{2}, \ldots, z_{n-1}\right\|\right)\right]^{p_{j}} \\
& \quad<\sup _{k}\left(\sum_{j=1}^{\infty} a_{k j}\right) \max \left\{\varepsilon_{1}^{G}, \varepsilon_{1}^{G_{0}}\right\}<\varepsilon \\
& \Longrightarrow \sum_{j=1}^{\infty} a_{k j}\left[M_{j} M_{j}^{\prime}\left(\left\|\frac{\Delta_{m}^{s}\left(\Lambda_{j}(x)\right)}{\rho_{1}}, z_{1}, z_{2}, \ldots, z_{n-1}\right\|\right)\right]^{p_{j}} \\
& \quad<\varepsilon .
\end{aligned}
$$

This shows that

$$
\begin{aligned}
& \{k \in \mathbb{N}: \\
& \sum_{j=1}^{\infty} a_{k j}\left[M _ { j } M _ { j } ^ { \prime } \left(\| \frac{\Delta_{m}^{s}\left(\Lambda_{j}(x)\right)}{\rho_{1}},\right.\right. \\
& \geq \varepsilon\} \subseteq A_{\delta} \in I .
\end{aligned}
$$

This proves the assertion. (b) Let $x=\left(x_{k}\right)$ be any element in $W\left[A, \mathscr{M}^{\prime}, \Lambda, p, \| \cdot \ldots\right.$. $\|]_{0}^{I}$. Then, by the following inequality, the results follow:

$$
\begin{aligned}
\sum_{j=1}^{\infty} a_{k j}\left[\left(M_{j}^{\prime}+M_{j}^{\prime \prime}\right)\left(\left\|\frac{\Delta_{m}^{s}\left(\Lambda_{j}(x)\right)}{\rho_{1}}, z_{1}, z_{2}, \ldots, z_{n-1}\right\|\right)\right]^{p_{j}} \\
\leq D \sum_{j=1}^{\infty} a_{k j}\left[M_{j}^{\prime}\left(\left\|\frac{\Delta_{m}^{s}\left(\Lambda_{j}(x)\right)}{\rho_{1}}, z_{1}, z_{2}, \ldots, z_{n-1}\right\|\right)\right]^{p_{j}} \\
+D \sum_{j=1}^{\infty} a_{k j}\left[M_{j}^{\prime \prime}\left(\left\|\frac{\Delta_{m}^{s}\left(\Lambda_{j}(x)\right)}{\rho_{1}}, z_{1}, z_{2}, \ldots, z_{n-1}\right\|\right)\right]^{p_{j}} .
\end{aligned}
$$

Theorem 13. The inclusions $Z\left[A, \mathscr{M}, \Delta_{m}^{s-1}, \Lambda,\|\cdot \ldots \cdot\|\right] \subseteq$ $Z\left[A, \mathscr{M}, \Delta_{m}^{s}, \Lambda,\|\cdot \ldots \cdot\|\right]$ are strict for $s, m \geq 1$ in general where $Z=W^{I}, W_{0}^{I}$, and $W_{\infty}^{I}$.

Proof. We will give the proof for $W\left[A, \mathscr{M}, \Delta_{m}^{s-1}, \Lambda,\|\cdot \ldots \cdot\|\right]_{0}^{I} \subseteq$ $W\left[A, \mathscr{M}, \Delta_{m}^{s}, \Lambda,\|\cdot \ldots \cdot\|\right]_{0}^{I}$ only. The others can be proved by similar arguments. Let $x=\left(x_{k}\right) \in W\left[A, \mathscr{M}, \Delta_{m}^{s-1}, \Lambda,\|\cdot \ldots \cdot\|\right]_{0}^{I}$. Then let $\varepsilon>0$ be given; there exist $\rho>0$ such that

$$
\begin{gathered}
\left\{k \in \mathbb{N}: \sum_{j=1}^{\infty} a_{k j} M_{j}\left(\left\|\frac{\Delta_{m}^{s-1}\left(\Lambda_{j}(x)\right)}{\rho}, z_{1}, z_{2}, \ldots, z_{n-1}\right\|\right)\right. \\
\left.\geq \frac{\varepsilon}{2}\right\} \in I .
\end{gathered}
$$

Since $M_{j}$ for all $j \in \mathbb{N}$ is non-decreasing and convex, it follows that

$$
\begin{aligned}
& \sum_{j=1}^{\infty} a_{k j} M_{j}\left(\left\|\frac{\Delta_{m}^{s}\left(\Lambda_{j}(x)\right)}{2 \rho}, z_{1}, z_{2}, \ldots, z_{n-1}\right\|\right) \\
& =\sum_{j=1}^{\infty} a_{k j} \\
& \quad \times M_{j}\left(\| \frac{\Delta_{m}^{s-1} \Lambda_{j+1}(x)-\Delta_{m}^{s-1}\left(\Lambda_{j}(x)\right)}{2 \rho},\right. \\
& \leq \frac{1}{2} \sum_{j=1}^{\infty} a_{k j} M_{j}\left(\left\|\frac{\Delta_{m}^{s-1}\left(\Lambda_{j+1}(x)\right)}{\rho}, z_{1}, z_{2}, \ldots, z_{n-1}\right\|\right) \\
& \quad+\frac{1}{2} \sum_{j=1}^{\infty} a_{k j} M_{j}\left(\left\|\frac{\Delta_{m}^{s-1}\left(\Lambda_{j}(x)\right)}{\rho}, z_{1}, z_{2}, \ldots, z_{n-1}\right\|\right)
\end{aligned}
$$


and then we have

$$
\begin{aligned}
& \left\{k \in \mathbb{N}: \sum_{j=1}^{\infty} a_{k j}\right. \\
& \times M_{j}\left(\| \frac{\Delta_{m}^{s}\left(\Lambda_{j}(x)\right)}{2 \rho},\right. \\
& \left.z_{1}, z_{2}, \ldots, z_{n-1} \| \geq \varepsilon\right\} \\
& \subseteq\left\{k \in \mathbb{N}: \frac{1}{2}\right. \\
& \times \sum_{j=1}^{\infty} a_{k j} M_{j}\left(\| \frac{\Delta_{m}^{s-1} x_{j+1}}{\rho},\right. \\
& \left.\left.z_{1}, z_{2}, \ldots, z_{n-1} \|\right) \geq \frac{\varepsilon}{2}\right\} \\
& \cup\left\{k \in \mathbb{N}: \frac{1}{2}\right. \\
& \times \sum_{j=1}^{\infty} a_{k j} M_{j}\left(\| \frac{\Delta_{m}^{s-1}\left(\Lambda_{j}(x)\right)}{\rho},\right. \\
& \left.\left.z_{1}, z_{2}, \ldots, z_{n-1} \|\right) \geq \frac{\varepsilon}{2}\right\} .
\end{aligned}
$$

Let $M_{k}(x)=M(x)=x$ for all $x \in[0, \infty[, k \in \mathbb{N}$ and $\lambda_{k}=k$ for all $k \in \mathbb{N}$. Consider a sequence $x=\left(x_{k}\right)=\left(k^{s}\right)$. Then, $x \in W\left[A, \mathscr{M}, \Delta_{m}^{s}, \Lambda,\|\cdot \ldots \cdot\|\right]_{0}^{I}$ but does not belong to $W\left[A, \mathscr{M}, \Delta_{m}^{s-1}, \Lambda,\|\cdot \ldots \cdot\|\right]_{0}^{I}$, for $s=m=1$. This shows that the inclusion is strict.

Theorem 14. Let $0<p_{k} \leq q_{k}$ for all $k \in \mathbb{N}$; then

$$
\begin{aligned}
& W\left[A, \mathscr{M}, \Delta_{m}^{s}, \Lambda, p,\|\cdot \ldots \cdot\|\right]_{\infty} \\
& \quad \subseteq W\left[A, \mathscr{M}, \Delta_{m}^{s}, \Lambda, q,\|\cdot \ldots \cdot\|\right]_{\infty} .
\end{aligned}
$$

Proof. Let $x=\left(x_{j}\right) \in W\left[A, \mathscr{M}, \Delta_{m}^{s}, \Lambda, p,\|\cdot \ldots \cdot\|\right]_{\infty}$; then there exists some $\rho>0$ such that

$$
\sup _{k} \sum_{j=1}^{\infty} a_{k j}\left[M_{j}\left(\left\|\frac{\Delta_{m}^{s}\left(\Lambda_{j}(x)\right)}{\rho}, z_{1}, z_{2}, \ldots, z_{n-1}\right\|\right)\right]^{p_{j}}<\infty .
$$

This implies that

$$
M_{j}\left(\left\|\frac{\Delta_{m}^{s}\left(\Lambda_{j}(x)\right)}{\rho}, z_{1}, z_{2}, \ldots, z_{n-1}\right\|\right)<1,
$$

for a sufficiently large value of $j$. Since $M_{j}$ for all $j \in \mathbb{N}$ is non-decreasing, we get

$$
\begin{aligned}
& \sup _{k} \sum_{j=1}^{\infty} a_{k j}\left[M_{j}\left(\left\|\frac{\Delta_{m}^{s}\left(\Lambda_{j}(x)\right)}{\rho}, z_{1}, z_{2}, \ldots, z_{n-1}\right\|\right)\right]^{q_{j}} \\
& \leq \sup _{k} \sum_{j=1}^{\infty} a_{k j}\left[M_{j}\left(\left\|\frac{\Delta_{m}^{s}\left(\Lambda_{j}(x)\right)}{\rho}, z_{1}, z_{2}, \ldots, z_{n-1}\right\|\right)\right]^{p_{j}}<\infty .
\end{aligned}
$$

Thus, $x \in W\left[A, \mathscr{M}, \Delta_{m}^{s}, \Lambda, q,\|\cdot \ldots \cdot\|\right]_{\infty}$. This completes the proof of the theorem.

Theorem 15. (i) If $0<\inf p_{k} \leq p_{k}<1$, then

$$
\begin{aligned}
& W\left[A, \mathscr{M}, \Delta_{m}^{s}, \Lambda, p,\|\cdot \ldots \cdot\|\right]_{\infty} \\
& \quad \subseteq W\left[A, \mathscr{M}, \Delta_{m}^{s}, \Lambda,\|\cdot \ldots \cdot\|\right]_{\infty} .
\end{aligned}
$$

(ii) If $1<p_{k} \leq \sup _{k} p_{k}<\infty$, then $W\left[A, \mathscr{M}, \Delta_{m}^{s}, \Lambda, \| \cdot \ldots\right.$. $\|]_{\infty} \subseteq W\left[A, \mathscr{M}, \Delta_{m}^{s}, \Lambda, p,\|\cdot \ldots \cdot\|\right]_{\infty}$.

Proof. (i) Let $x=\left(x_{j}\right) \in W\left[A, \mathscr{M}, \Delta_{m}^{s}, \Lambda, p,\|\cdot \ldots \cdot\|\right]_{\infty}$; since $0<\inf _{k} p_{k} \leq p_{k}<1$, then we have

$$
\begin{aligned}
& \sup _{k} \sum_{j=1}^{\infty} a_{k j}\left[M_{j}\left(\left\|\frac{\Delta_{m}^{s}\left(\Lambda_{j}(x)\right)}{\rho}, z_{1}, z_{2}, \ldots, z_{n-1}\right\|\right)\right] \\
& \leq \sup _{k} \sum_{j=1}^{\infty} a_{k j}\left[M_{j}\left(\left\|\frac{\Delta_{m}^{s}\left(\Lambda_{j}(x)\right)}{\rho}, z_{1}, z_{2}, \ldots, z_{n-1}\right\|\right)\right]^{p_{j}}<\infty,
\end{aligned}
$$

and hence $x \in W\left[A, \mathscr{M}, \Delta_{m}^{s}, \Lambda,\|\cdot \ldots \cdot\|\right]_{\infty}$.

(ii) Let $1<p_{k} \leq \sup _{k} p_{k}<\infty$ and $x=\left(x_{j}\right) \epsilon$ $W\left[A, \mathscr{M}, \Delta_{m}^{s}, \Lambda,\|\cdot \ldots \cdot\|\right]_{\infty}$. Then for each $0<\varepsilon<1$ there exists a positive integer $j_{0}$ such that

$$
\begin{aligned}
& \sup _{k} \sum_{j=1}^{\infty} a_{k j}\left[M_{j}\left(\left\|\frac{\Delta_{m}^{s}\left(\Lambda_{j}(x)\right)}{\rho}, z_{1}, z_{2}, \ldots, z_{n-1}\right\|\right)\right] \\
& \quad \leq \varepsilon<1,
\end{aligned}
$$

for all $j \geq j_{0}$. This implies that

$$
\begin{aligned}
& \sup _{k} \sum_{j=1}^{\infty} a_{k j}\left[M_{j}\left(\left\|\frac{\Delta_{m}^{s}\left(\Lambda_{j}(x)\right)}{\rho}, z_{1}, z_{2}, \ldots, z_{n-1}\right\|\right)\right]^{p_{j}} \\
& \leq \sup _{k} \sum_{j=1}^{\infty} a_{k j}\left[M_{j}\left(\left\|\frac{\Delta_{m}^{s}\left(\Lambda_{j}(x)\right)}{\rho}, z_{1}, z_{2}, \ldots, z_{n-1}\right\|\right)\right]<\infty .
\end{aligned}
$$

Thus $x \in W\left[A, \mathscr{M}, \Delta_{m}^{s}, \Lambda, p,\|\cdot \ldots \cdot\|\right]_{\infty}$ and this completes the proof.

Theorem 16. For any sequence of the Orlicz functions $M=\left(M_{j}\right)$ which satisfies the $\Delta_{2}$-condition, we have $W\left[A, \Delta_{m}^{s}, \Lambda, p,\|\cdot \ldots \cdot\|\right]^{I} \subset W\left[A, \mathscr{M}, \Delta_{m}^{s}, \Lambda, p,\|\cdot \ldots \cdot\|\right]^{I}$. 
Proof. Let $x=\left(x_{j}\right) \in W\left[A, \Delta_{m}^{s}, \Lambda, p,\|\cdot \ldots \cdot\|\right]^{I}$ and $\varepsilon>0$ be given. Then, there exist $\rho>0$ such that the set

$$
\begin{aligned}
& \{k \in \mathbb{N}: \\
& \sum_{j=1}^{\infty} a_{k j}\left[\| \frac{\Delta_{m}^{s}\left(\Lambda_{j}(x)\right)-l}{\rho},\right. \\
& \left.\left.z_{1}, z_{2}, \ldots, z_{n-1} \|\right]^{p_{j}} \geq \varepsilon\right\}
\end{aligned}
$$

$\in I$, for some $l$.

By taking $y_{j}=\left\|\left(\Delta_{m}^{s}\left(\Lambda_{j}(x)\right)-l\right) / \rho, z_{1}, z_{2}, \ldots, z_{n-1}\right\|$, let $\varepsilon>0$ and choose $\delta$ wit $0<\delta<1$ such that $M_{j}(t)<\varepsilon$ for all $j \in \mathbb{N}$; for $0 \leq t \leq \delta$, consider that

$$
\begin{aligned}
\sum_{j=1}^{\infty} & {\left[M_{j}\left(y_{j}\right)\right]^{p_{j}} } \\
= & \sum_{j=1, y_{j} \leq \delta}^{\infty}\left[M_{j}\left(y_{j}\right)\right]^{p_{j}} \\
& +\sum_{j=1, y_{j}>\delta}^{\infty}\left[M_{j}\left(y_{j}\right)\right]^{p_{j}},
\end{aligned}
$$

since $M_{j}$ is continuous for all $n \in \mathbb{N}$.

$\sum_{j \in I_{k}, y_{j} \leq \delta}\left[M_{j}\left(y_{j}\right)\right]^{p_{j}}<\varepsilon$ and for $y_{j}>\delta$, we use the fact that $y_{j}<y_{j} / \delta<1+y_{j} / \delta$. Since $\mathscr{M}=\left(M_{j}\right)$ is non-decreasing and convex, it follows that

$$
M_{j}\left(y_{j}\right)<M_{j}\left(1+\frac{y_{j}}{\delta}\right)<\frac{1}{2} M_{j}(2)+\frac{1}{2} M_{j}\left(\frac{2 y_{j}}{\delta}\right) .
$$

Since $\mathscr{M}=\left(M_{j}\right)$ satisfies the $\Delta_{2}$-condition, then

$$
M_{j}\left(y_{j}\right)<\frac{y_{j}}{2 \delta} L M_{j}(2)+\frac{y_{j}}{2 \delta} L M_{j}(2)=\frac{y_{j}}{\delta} L M_{j}(2) .
$$

Hence

$$
\begin{aligned}
& \sum_{j=1, y_{j}>\delta}^{\infty}\left[M_{j}\left(y_{j}\right)\right]^{p_{j}} \\
& <\max \left\{1, \sup _{j}\left(L \delta^{-1} M_{j}(2)\right)^{p_{j}}\right\} \\
& \quad \times \sum_{j=1, y_{j}>\delta}^{\infty}\left(y_{j}\right)^{p_{j}},
\end{aligned}
$$

and then we have

$$
\begin{aligned}
& \sum_{j=1}^{\infty}\left[M_{j}\left(y_{j}\right)\right]^{p_{j}} \\
& \quad<\varepsilon+\max \left\{1, \sup _{j}\left(L \delta^{-1} M_{j}(2)\right)^{p_{j}}\right\} \sum_{j=1, y_{j}>\delta}^{\infty}\left(y_{j}\right)^{p_{j}} .
\end{aligned}
$$

This proves that $W\left[A, \Delta_{m}^{s}, \Lambda, p, \| \cdot \ldots \cdot\right]^{I} \quad \subset \quad$ $W\left[A, \mathscr{M}, \Delta_{m}^{s}, \Lambda, p,\|\cdot \ldots \cdot\|\right]^{I}$.

Theorem 17. Let $0<p_{n} \leq q_{n}<1$ and $\left(q_{n} / p_{n}\right)$ be bounded; then

$$
\begin{aligned}
& W\left[A, \mathscr{M}, \Delta_{m}^{s}, \Lambda, q,\|\cdot \ldots \cdot\|\right]^{I} \\
& \quad \subseteq W\left[A, \mathscr{M}, \Delta_{m}^{s}, \Lambda, p,\|\cdot \ldots \cdot\|\right]^{I} .
\end{aligned}
$$

Proof. Let $x=\left(x_{j}\right) \in W[A, \mathscr{M}, \Lambda, q,\|\cdot \ldots \cdot\|]_{\infty}$ and we put

$$
\begin{gathered}
y_{j}=\left[M_{j}\left(\left\|\frac{\Delta_{m}^{s}\left(\Lambda_{j}(x)\right)-l}{\rho}, z_{1}, z_{2}, \ldots, z_{n-1}\right\|\right)\right]^{q_{j}}, \\
\beta_{j}=\frac{p_{j}}{q_{j}} \quad \forall j \in \mathbb{N} .
\end{gathered}
$$

Then $0<\beta_{j} \leq 1$, for all $j \in \mathbb{N}$. Let it be such that $0<$ $\beta \leq \beta_{j}$ for all $j \in \mathbb{N}$. Define the sequences $\left(a_{j}\right)$ and $\left(b_{j}\right)$ as follows: for $y_{j} \geq 1$, let $a_{j}=y_{j}$ and $b_{j}=0$; for $y_{j}<1$, let $a_{j}=$ 0 and $b_{j}=y_{j}$. Then clearly, for all $j \in \mathbb{N}$ we have $y_{j}=a_{j}+$ $b_{j}, y_{j}^{\beta_{j}}=a_{j}^{\beta_{j}}+b_{j}^{\beta_{j}}, a_{j}^{\beta_{j}} \leq a_{j} \leq y_{j}$, and $b_{j}^{\beta_{j}} \leq b_{j}^{\beta}$. Therefore, we have

$$
\sum_{j=1}^{\infty} a_{k j} y_{j}^{\beta_{j}} \leq \sum_{j=1}^{\infty} a_{k j} y_{j} \leq\left[\sum_{j=1}^{\infty} a_{k j} y_{j}\right]^{\beta} .
$$

Hence $x \in W\left[A, \mathscr{M}, \Delta_{m}^{s}, \Lambda, p,\|\cdot \ldots \cdot\|\right]_{\infty}$.

Theorem 18. For any two sequences $p=\left(p_{k}\right)$ and $q=$ $\left(q_{k}\right)$ of positive real numbers and for any two $n$ norms $\|\cdot \ldots \cdot\|_{1}$ and $\|\cdot \ldots \cdot\|_{2}$ on $X$, the following holds:

$$
\begin{aligned}
& Z\left[A, \mathscr{M}, \Delta_{m}^{s}, \Lambda, p,\|\cdot \ldots \cdot\|_{1}\right] \\
& \cap Z\left[A, \mathscr{M}, \Delta_{m}^{s}, \Lambda, q,\|\cdot \ldots \cdot\|_{2}\right] \neq \phi
\end{aligned}
$$

where $Z=W^{I}, W_{0}^{I}, W_{\infty}^{I}$, and $W_{\infty}$.

Proof. The proof of the theorem is obvious, because the zero element belongs to each of the sequence spaces involved in the intersection.

Theorem 19. The sequence spaces $W\left[A, \mathscr{M}, \Delta_{m}^{s}, \Lambda, p, \| \cdot \ldots \cdot\right.$ $\|]^{I}, W\left[A, \mathscr{M}, \Delta_{m}^{s}, \Lambda, p,\|\cdot \ldots \cdot\|\right]_{0}^{I}, W\left[A, \mathscr{M}, \Delta_{m}^{s}, \Lambda, p, \| \cdot \ldots \cdot\right.$ $\|]_{\infty}$, and $W\left[A, \mathscr{M}, \Delta_{m}^{s}, \Lambda, p,\|\cdot \ldots \cdot\|\right]_{\infty}^{I}$ are neither solid nor symmetric nor sequence algebras for $s, m \geq 1$ in general.

Proof. The proof is obtained by using the same techniques of Et [23] and Theorems 15, 17, and 18.

Note 1. It is clear from definitions that

$$
\begin{aligned}
& W\left[A, \mathscr{M}, \Delta_{m}^{s}, \Lambda, p,\|\cdot \ldots \cdot\|\right]_{0}^{I} \\
& \quad \subseteq W\left[A, \mathscr{M}, \Delta_{m}^{s}, \Lambda, p,\|\cdot \ldots \cdot\|\right]^{I} \\
& \quad \subseteq W\left[A, \mathscr{M}, \Delta_{m}^{s}, \Lambda, p,\|\cdot \ldots \cdot\|\right]_{\infty}^{I} .
\end{aligned}
$$


Theorem 20. The spaces $Z\left[A, \mathscr{M}, \Delta_{m}^{s}, \Lambda, p,\|\cdot \ldots \cdot\|\right]$ and $Z[A, \mathscr{M}, p,\|\cdot \ldots \cdot\|]$ are equivalent as topological spaces, where $Z=W^{I}, W_{0}^{I}, W_{\infty}^{I}$, and $W_{\infty}$.

Proof. Consider the mapping

$$
T: Z\left[A, \mathscr{M}, \Delta_{m}^{s}, \Lambda, p,\|\cdot \ldots \cdot\|\right] \longrightarrow Z[A, \mathscr{M}, p,\|\cdot \ldots \cdot\|]
$$

defined by $T(x)=\left(\Delta_{m}^{s}\left(\Lambda_{j} x\right)\right)$ for each $x \in$ $Z\left[A, \mathscr{M}, \Delta_{m}^{s}, \Lambda, p,\|\cdot \ldots \cdot\|\right]$. Then, clearly $T$ is a linear homeomorphism and the proof follows.

Remark 21. If we replace the difference operator $\Delta_{m}^{s}$ by $\Delta_{m}^{(s)}$, then for each $\varepsilon>0$ we get the following sequence spaces:

$$
\begin{aligned}
& W\left[A, \mathscr{M}, \Delta_{m}^{(s)}, \Lambda, p,\|\cdot \ldots \cdot\|\right]^{I} \\
& =\{x \in \omega(n-X): \\
& \{k \in \mathbb{N}: \\
& \lambda_{k}^{-1} \sum_{j \in I_{k}}\left[M _ { j } \left(\| \frac{\Delta_{m}^{(s)} x_{j}-l}{\rho},\right.\right. \\
& \left.\left.z_{1}, z_{2}, \ldots, z_{n-1} \|\right)\right]^{p_{j}} \\
& \geq \varepsilon\} \in I,
\end{aligned}
$$

for some $\rho>0, l \in X$ and each

$$
\begin{gathered}
\left.z_{1}, z_{2}, \ldots, z_{n-1} \in X\right\}, \\
W\left[A, \mathscr{M}, \Delta_{m}^{(s)}, \Lambda, p,\|\cdot \ldots \cdot\|\right]_{0}^{I} \\
=\{x \in \omega(n-X): \\
\left\{\begin{array}{c}
k \in \mathbb{N}: \\
\lambda_{k}^{-1} \sum_{j \in I_{k}}\left[M _ { j } \left(\| \frac{\Delta_{m}^{(s)} x_{j}}{\rho},\right.\right.
\end{array}\right. \\
\left.\left.\qquad z_{1}, z_{2}, \ldots, z_{n-1} \|\right)\right]^{p_{j}}
\end{gathered}
$$$$
\geq \varepsilon\} \in I
$$

for some $\rho>0$ and each $\left.z_{1}, z_{2}, \ldots, z_{n-1} \in X\right\}$, $W\left[A, \mathscr{M}, \Delta_{m}^{(s)}, \Lambda, p,\|\cdot \ldots \cdot\|\right]_{\infty}$

$$
=\{x \in \omega(n-X):
$$

$$
\begin{aligned}
& \sup _{k} \lambda_{k}^{-1} \sum_{j \in I_{k}}\left[M_{j}\left(\left\|\frac{\Delta_{m}^{(s)} x_{j}}{\rho}, z_{1}, z_{2}, \ldots, z_{n-1}\right\|\right)\right]^{p_{j}} \\
& <\infty
\end{aligned}
$$

for some $\rho>0$ and each $\left.z_{1}, z_{2}, \ldots, z_{n-1} \in X\right\}$,

$$
\begin{aligned}
& W\left[A, \mathscr{M}, \Delta_{m}^{(s)}, \Lambda, p,\|\cdot \ldots \cdot\|\right]_{\infty}^{I} \\
& =\{x \in \omega(n-X): \exists K>0, \\
& \quad \text { s.t. }\left\{k \in \mathbb{N}: \lambda_{k}^{-1} \sum_{j \in I_{k}}\left[M _ { j } \left(\| \frac{\Delta_{m}^{(s)} x_{j}}{\rho},\right.\right.\right. \\
& \geq K\} \in I, \\
& \text { for some } \left.\rho>0 \text { and each } z_{1}, z_{2}, \ldots, z_{n-1} \in X\right\} .
\end{aligned}
$$

Corollary 22. The sequence spaces $Z\left[A, \mathscr{M}, \Delta_{m}^{s}, \Lambda, p, \|\right.$. $\ldots \cdot \|]$, where $Z=W^{I}, W_{0}^{I}, W_{\infty}^{I}$, and $W_{\infty}$, are paranormed spaces (not totally paranormed) with respect to the paranorm $h_{\Delta}$ defined by

$$
\begin{aligned}
h_{\Delta}(x)= & \sum_{j=1}^{m s}\left\|x_{j}, z_{1}, z_{2}, \ldots, z_{n-1}\right\| \\
& +\inf \left\{\rho^{p_{k} / H}:\right.
\end{aligned}
$$




$$
\begin{aligned}
\sup _{k}\left[\sum_{j=1}^{\infty} a_{k j}\right. & \\
\times & {\left[M _ { j } \left(\| \frac{\Delta_{m}^{(s)}\left(\Lambda_{j}(x)\right)}{\rho_{1}},\right.\right.} \\
& \left.\left.\left.z_{1}, z_{2}, \ldots, z_{n-1} \|\right)\right]^{p_{j}}\right]^{1 / H}
\end{aligned}
$$

$\leq 1$, for some $\rho>0$,

and each $\left.z_{1}, z_{2}, \ldots, z_{n-1} \in X\right\}$,

where $H=\max \left\{1, \sup _{k} p_{k}\right\}$ and $Z=W^{I}, W_{0}^{I}, W_{\infty}^{I}$, and $W_{\infty}$. Also it is clear that the paranorms $g_{\Delta}$ and $h_{\Delta}$ are equivalent.

We state the following theorem in view of Lemma 9.

Theorem 23. Let $X$ be a standard n-normed space and $\left\{e_{1}, e_{2}, \ldots, e_{n}\right\}$ an orthogonal set in $X$. Then, the following hold:

(a) $W\left[A, \mathscr{M}, \Delta_{m}^{(s)}, \Lambda, p,\|\cdot \ldots \cdot\|_{\infty}\right]^{I}=W\left[A, \mathscr{M}, \Delta_{m}^{(s)}, \Lambda\right.$, $\left.p,\|\cdot \ldots \cdot\|_{n-1}\right]^{I}$,

(b) $W\left[A, \mathscr{M}, \Delta_{m}^{(s)}, \Lambda, p,\|\cdot \ldots \cdot\|_{\infty}\right]_{0}^{I}=W\left[A, \mathscr{M}, \Delta_{m}^{(s)}, \Lambda\right.$, $\left.p,\|\cdot \ldots \cdot\|_{n-1}\right]_{0}^{I}$,

(c) $W\left[A, \mathscr{M}, \Delta_{m}^{(s)}, \Lambda, p,\|\cdot \ldots \cdot\|_{\infty}\right]_{\infty}=W\left[A, \mathscr{M}, \Delta_{m}^{(s)}, \Lambda\right.$, $\left.p,\|\cdot \ldots \cdot\|_{n-1}\right]_{\infty}$

(d) $W\left[A, \mathscr{M}, \Delta_{m}^{(s)}, \Lambda, p,\|\cdot \ldots \cdot\|_{\infty}\right]_{\infty}^{I}=W\left[A, \mathscr{M}, \Delta_{m}^{(s)}, \Lambda\right.$, $\left.p,\|\cdot \ldots \cdot\|_{n-1}\right]_{\infty}^{I}$

where $\|\cdot \ldots \cdot\|_{\infty}$ is the derived $(n-1)$-norm defined with respect to the set $\left\{e_{1}, e_{2}, \ldots, e_{n}\right\}$ and $\|\cdot \ldots \cdot\|_{n-1}$ is the standard $(n-1)$-norm on $X$.

\section{Acknowledgment}

The author is most grateful to the editor and anonymous referee for careful reading of the paper and valuable suggestions which helped in improving an earlier version of it.

\section{References}

[1] P. Kostyrko, T. Šalàt, and W. Wilczyński, "On I-convergence," Real Analysis Exchange, vol. 26, no. 2, pp. 669-685, 2000-2001.

[2] S. Gähler, "Linear 2-normietre Räume," Mathematische Nachrichten, vol. 28, pp. 1-43, 1965.

[3] A. Misiak, " $n$-inner product spaces," Mathematische $\mathrm{Na}$ chrichten, vol. 140, no. 1, pp. 299-319, 1989.

[4] H. Gunawan, "The space of $p$-summable sequences and its natural $n$-norm," Bulletin of the Australian Mathematical Society, vol. 64, no. 1, pp. 137-147, 2001.
[5] H. Gunawan and M. Mashadi, “On n-normed spaces," International Journal of Mathematics and Mathematical Sciences, vol. 27, no. 10, pp. 631-639, 2001.

[6] H. Gunawan and M. Mashadi, "On finite-dimensional 2normed spaces," Soochow Journal of Mathematics, vol. 27, no. 3, pp. 147-169, 2001.

[7] E. Savaş, " $\Delta^{m}$-strongly summable sequences spaces in 2-normed spaces defined by ideal convergence and an Orlicz function," Applied Mathematics and Computation, vol. 217, no. 1, pp. 271-276, 2010.

[8] M. Gürdal, "On ideal convergent sequences in 2-normed spaces," Thai Journal of Mathematics, vol. 4, no. 1, pp. 85-91, 2006.

[9] M. Gürdal and A. Şahiner, "New sequence spaces in $n$-normed spaces with respect to an Orlicz function," The Aligarh Bulletin of Mathematics, vol. 27, no. 1, pp. 53-58, 2008.

[10] M. Mursaleen and A. K. Noman, "On the spaces of $\lambda$-convergent and bounded sequences," Thai Journal of Mathematics, vol. 8, no. 2, pp. 311-329, 2010.

[11] H. Nakano, "Concave modulars," Journal of the Mathematical Society of Japan, vol. 5, pp. 29-49, 1953.

[12] W. H. Ruckle, "FK spaces in which the sequence of coordinate vectors is bounded," Canadian Journal of Mathematics, vol. 25, pp. 973-978, 1973.

[13] I. J. Maddox, "Sequence spaces defined by a modulus," Mathematical Proceedings of the Cambridge Philosophical Society, vol. 100, no. 1, pp. 161-166, 1986.

[14] J. Lindenstrauss and L. Tzafriri, "On Orlicz sequence spaces," Israel Journal of Mathematics, vol. 10, pp. 379-390, 1971.

[15] M. Gungor and M. Et, " $\Delta^{r}$-strongly almost summable sequences defined by Orlicz functions," Indian Journal of Pure and Applied Mathematics, vol. 34, no. 8, pp. 1141-1151, 2003.

[16] M. Et, Y. Altin, B. Choudhary, and B. C. Tripathy, "On some classes of sequences defined by sequences of Orlicz functions," Mathematical Inequalities \& Applications, vol. 9, no. 2, pp. 335342, 2006.

[17] B. C. Tripathy, Y. Altin, and M. Et, "Generalized difference sequence spaces on seminormed space defined by Orlicz functions," Mathematica Slovaca, vol. 58, no. 3, pp. 315-324, 2008.

[18] H. Kizmaz, "On certain sequence spaces," Canadian Mathematical Bulletin, vol. 24, no. 2, pp. 169-176, 1981.

[19] M. Et and R. Colak, "On generalized difference sequence spaces," Soochow Journal of Mathematics, vol. 21, no. 4, pp. 377386, 1995.

[20] B. C. Tripathy and A. Esi, "A new type of sequence spaces," International Journal of Food Science \& Technology, vol. 1, no. 1, pp. 11-14, 2006.

[21] B. C. Tripathy, A. Esi, and B. K. Tripathy, "On a new type of generalized difference Cesáro sequence spaces," Soochow Journal of Mathematics, vol. 31, no. 3, pp. 333-340, 2005.

[22] A. A. Bakery, E. A. Elnour, and M.-A. Ahmed, "Some generalized difference sequence spaces defined by ideal convergence and Musielak-Orlicz function," Abstract and Applied Analysis, vol. 2013, Article ID 972363, 10 pages, 2013.

[23] M. Et, "Spaces of Cesàro difference sequences of order defined by a modulus function in a locally convex space," Taiwanese Journal of Mathematics, vol. 10, no. 4, pp. 865-879, 2006. 


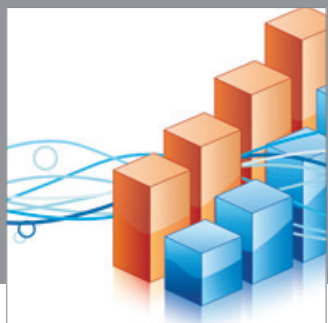

Advances in

Operations Research

mansans

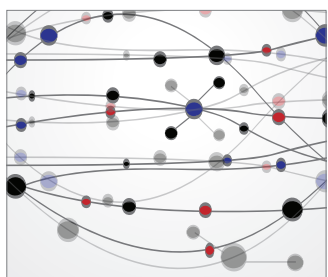

The Scientific World Journal
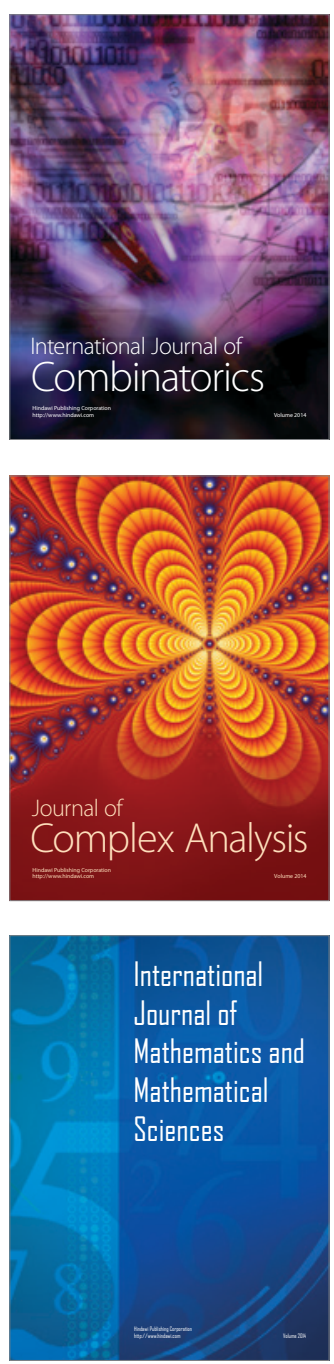
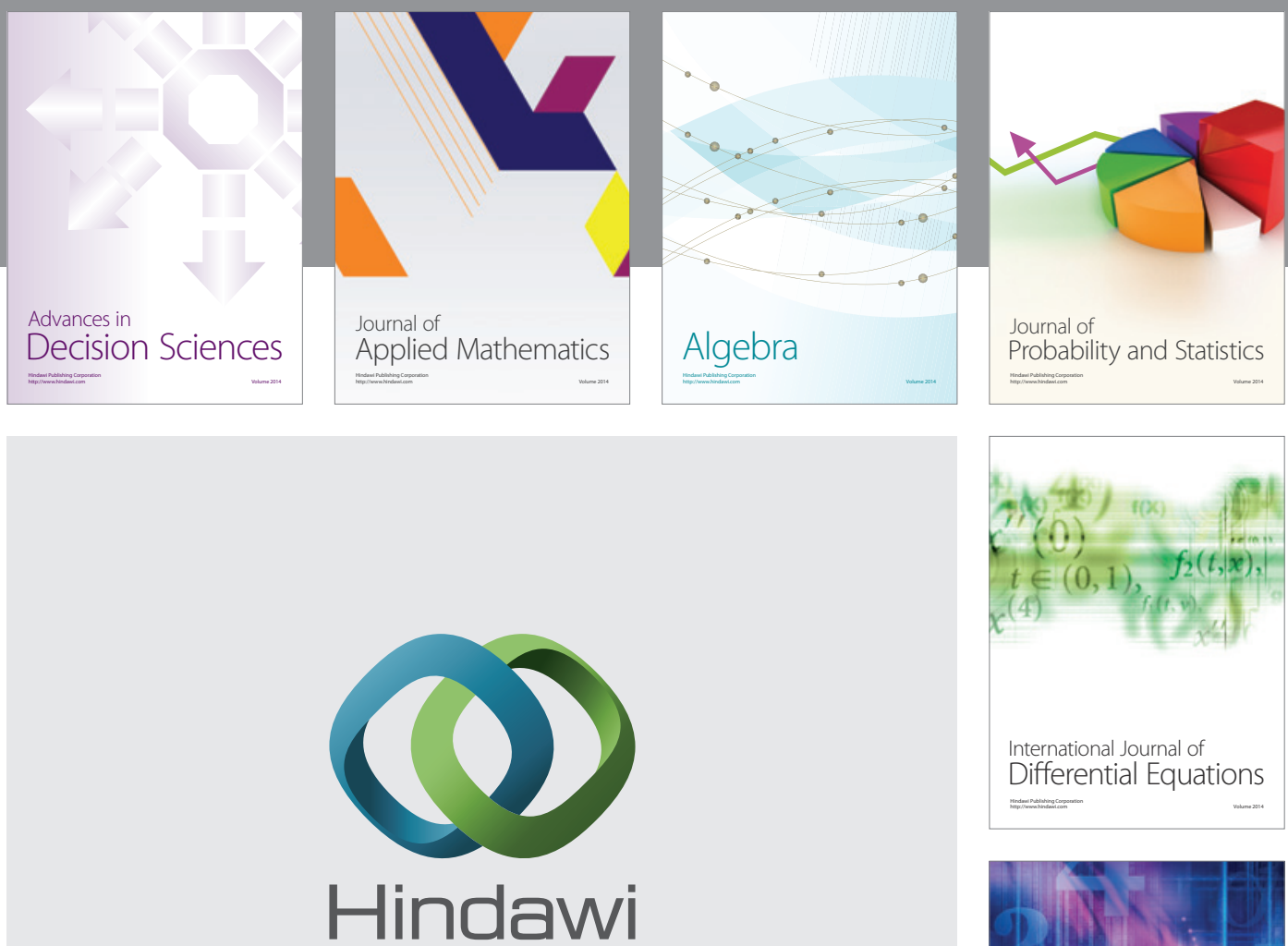

Submit your manuscripts at http://www.hindawi.com
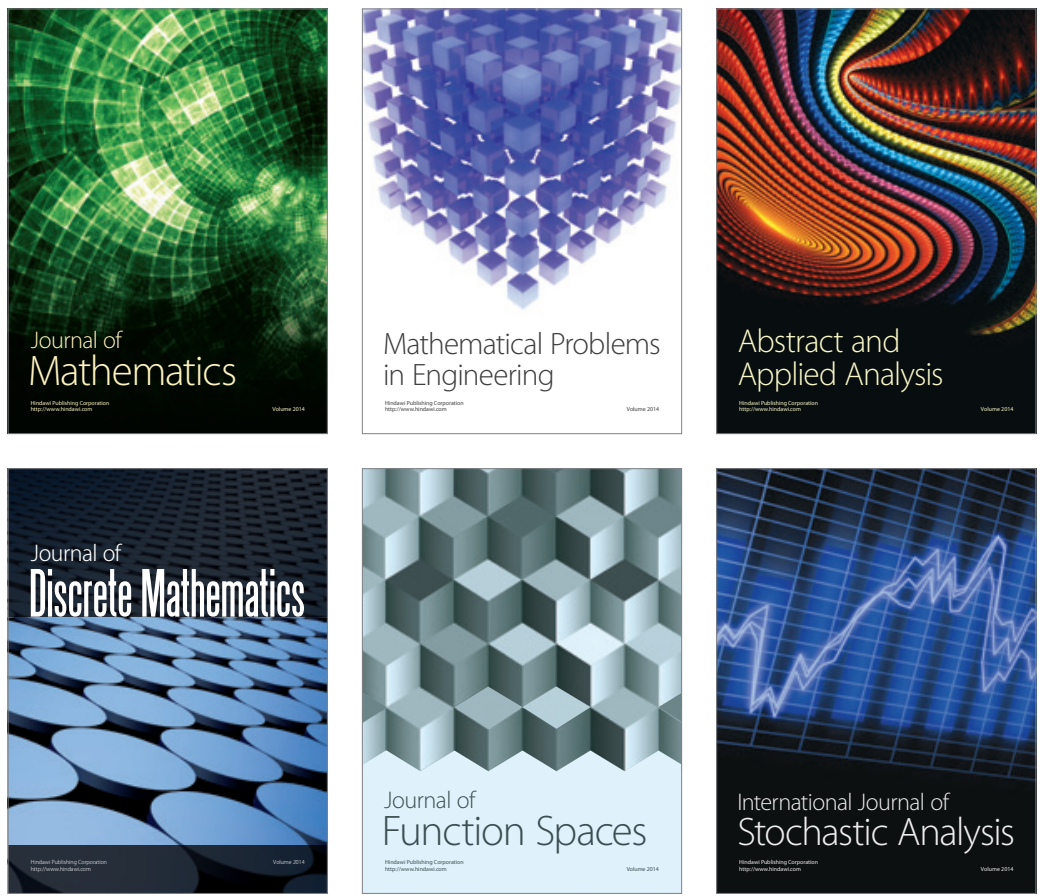

Journal of

Function Spaces

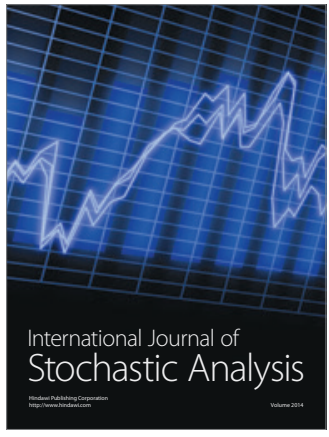

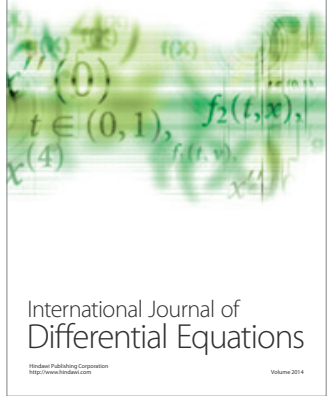
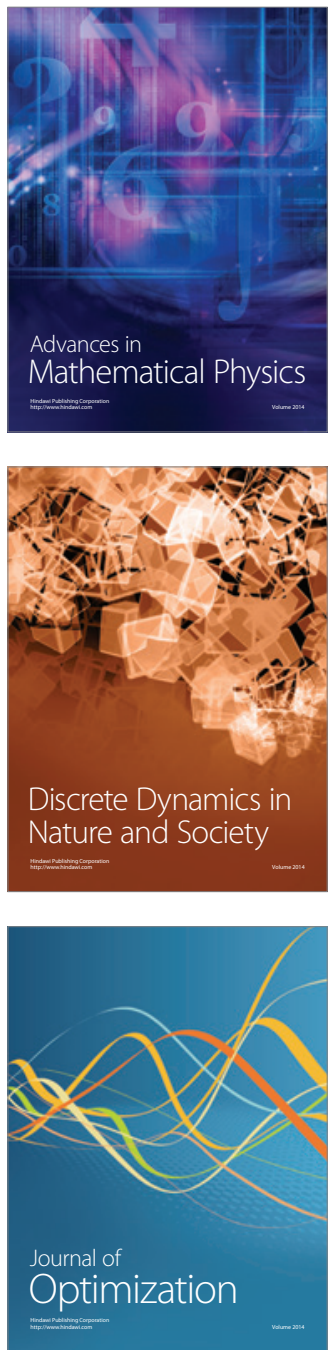